\title{
openheart Role of adjuvant carotid ultrasound in women undergoing stress echocardiography for the assessment of suspected coronary artery disease
}

\author{
Sothinathan Gurunathan (D) , ${ }^{1,2}$ Mayooran Shanmuganathan, ${ }^{1,2}$ Reinette Hampson, ${ }^{2}$ \\ Rajdeep Khattar, ${ }^{1,3}$ Roxy Senior (1) ${ }^{1,2,3}$
}

To cite: Gurunathan S, Shanmuganathan $\mathrm{M}$, Hampson R, et al. Role of adjuvant carotid ultrasound in women undergoing stress echocardiography for the assessment of suspected coronary artery disease. Open Heart 2020;7:e001188. doi:10.1136/

openhrt-2019-001188

Our work has been previously presented at the ESC Congress 2019 and published as a conference abstract https://doi. org/10.1093/eurheartj/ehz748. 0269.

Received 12 0ctober 2019 Revised 10 March 2020 Accepted 29 April 2020

Check for updates

(c) Author(s) (or their employer(s)) 2020. Re-use permitted under CC BY-NC. No commercial re-use. See rights and permissions. Published by BMJ.

${ }^{1}$ National Heart and Lung Institute, Imperial College London, London, UK ${ }^{2}$ Department of Cardiology, Northwick Park Hospital,

Harrow, UK

${ }^{3}$ Department of Cardiology, Royal Brompton and Harefield NHS Foundation Trust, London, UK

Correspondence to Professor Roxy Senior; roxysenior@cardiac-research. org

\section{ABSTRACT}

Objective Due to the low prevalence of obstructive coronary artery disease (CAD) in women, stress testing has a relatively low predictive value for this. Additionally, conventional cardiovascular risk scores underestimate risk in women. This study sought to evaluate the role of atherosclerosis assessment using carotid ultrasound (CU) in women attending for stress echocardiography (SE). Methods This was a prospective study in which consecutive women with recent-onset suspected angina, who were referred for clinically indicated SE, underwent CU.

Results 415 women (mean age $61 \pm 10$ years, 29\% diabetes mellitus, mean body mass index 28) attending for SE underwent CU. 47 women (11\%) had inducible wall motion abnormalities, and carotid disease (CD) was present in $46 \%$ (carotid plaque in $41 \%$, carotid intimamedia thickness $>75$ th percentile in $15 \%$ ). Women with CD were older (65 vs 58 years, $p<0.001$ ), and more likely to have diabetes ( $41 \%$ vs $21 \%, p=0.001$ ), hypertension ( $67 \%$ vs $36 \%, p<0.01)$ and a higher pretest probability of CAD ( $59 \%$ vs $41 \%, p<0.001) .40 \%$ of women classified as low Framingham risk were found to have evidence of CD. The positive predictive value of SE for flow-limiting CAD was $51 \%$, but with the presence of carotid plaque this was $71 \%(p<0.01)$. Carotid plaque $(p=0.004)$ and ischaemia $(p=0.01)$ were the only independent predictors of $>70 \%$ angiographic stenosis. In women with ischaemia on SE and no carotid plaque, the negative predictive value for flow-limiting disease was $88 \%$.

During a follow-up of $1058 \pm 234$ days, there were 15 events (defined as all-cause mortality, non-fatal myocardial infarction, heart failure admissions and late coronary revascularisation). Age (HR 1.07 (1.00-1.15), $\mathrm{p}=0.04)$, carotid plaque burden (HR $1.65(1.36-2.00)$, $\mathrm{p}<0.001)$ and ischaemic burden (HR $1.41(1.18-1.68)$, $\mathrm{p}<0.001)$ were associated with outcome. There was a stepwise increase in events/year from $0.3 \%$ when there were no ischaemia and atherosclerosis, $1.1 \%$ when there was atherosclerosis and no ischaemia, $2.2 \%$ when there was ischaemia and no atherosclerosis and $10 \%$ when there were both ischaemia and atherosclerosis $(p<0.001)$.

Conclusion CU significantly improves the accuracy of SE alone for identifying flow-limiting disease on coronary angiography, and improves risk stratification in women

\section{Key questions}

What is already known about this subject?

- The incremental diagnostic and prognostic value of carotid ultrasound (CU) to stress echocardiography (SE) in a general population has been previously described.

What does this study add?

- In view of the burden of cardiovascular disease in women and the need to improve outcomes, as well as the high false-positive rate with stress testing, a separate study in women was vital. In this study, $40 \%$ of women had subclinical atherosclerosis, who would have been discharged with no lifestyle or pharmacological intervention. Additionally, in women with inducible ischaemia on SE and no plaque on CU, the negative predictive value for flow-limiting coronary artery disease (CAD) was $88 \%$, suggesting that such patients should be referred for CT coronary angiography rather than invasive coronary angiography. The risk stratification with these complementary techniques was excellent with the event rate ranging from $0.3 \% /$ year (when both $\mathrm{CU}$ and SE were normal) to $10 \% /$ year (when both CU and SE were abnormal).

How might this impact on clinical practice?

- We would encourage the practice of assessing for carotid plaque, especially in women with a positive SE, in view of the high negative predictive value for obstructive CAD, where plaque is absent. In the absence of carotid plaque non-invasive angiography is recommended. Where plaque is identified, aggressive lifestyle modification and pharmacological intervention should be encouraged.

attending for SE, as well identifying a subset of women who may benefit from primary preventative measures.

\section{INTRODUCTION}

Ischaemic heart disease (IHD) is the leading cause of death in women of all ages, accounting for one-third of all female deaths 
globally. ${ }^{1}$ Coronary artery disease (CAD) mortality rates have increased in younger women aged 35-44 years. ${ }^{2}$ Resource consumption patterns in women are characterised by more frequent angina diagnosis, more clinic visits and hospitalisations, higher myocardial infarction (MI) mortality and higher heart failure rates as compared with men. ${ }^{3}$

There are several challenges for the clinician for the assessment of IHD in women: (1) the significant prevalence of non-obstructive $\mathrm{CAD}$ in women despite the presence of myocardial ischaemia; (2) the low prevalence of obstructive $\mathrm{CAD}$ reduces the positive predictive value (PPV) of functional testing; (3) cardiovascular risk assessment scores underestimate risk; (4) the poor outcomes necessitate accurate risk stratification and early intervention in high-risk groups; (5) the need to avoid ionising radiation where possible, particularly in younger women.

Stress echocardiography (SE) has excellent clinical utility in diverse populations and its lack of ionising radiation potentially makes it a suitable technique in women. ${ }^{4}$ However, the high prevalence of non-obstructive CAD results in a higher 'false-positive' rate for inducible wall motion abnormalities in women, which may result in unnecessary referral for invasive coronary angiography. ${ }^{5}$ Furthermore, the absence of myocardial ischaemia does not exclude non-obstructive $\mathrm{CAD}$, which has been shown to compromise prognosis. ${ }^{6}$ Carotid ultrasound (CU) has been shown be an excellent technique for the detection of atherosclerosis and has been shown to be an independent predictor of obstructive CAD as detected by coronary angiography. ${ }^{7}$ We have previously reported on the role of $\mathrm{CU}$ in a general population undergoing $\mathrm{SE}{ }^{8}$ However, due to the burden of cardiovascular disease in women and the need to improve outcomes, the unique pathophysiological mechanisms for ischaemia as well as the high false-positive rate with stress testing, a separate study in women is fundamental.

Thus, the primary hypothesis to be tested was that the performance of $\mathrm{CU}$ in addition to $\mathrm{SE}$ would reduce false-positive SE results for the prediction of CAD, and thus perform as a superior gatekeeper to invasive testing to SE alone. Furthermore, the absence of carotid atherosclerosis and myocardial ischaemia will confer a considerably lower risk status in symptomatic women with suspected angina. We also postulated that since myocardial ischaemia in women may occur in the absence of flow-limiting CAD or even in the absence of atherosclerosis, due to abnormal coronary reactivity or microvascular dysfunction, SE will identify high-risk patients.

Thus, the aim of this study was to prospectively assess the role of atherosclerosis imaging with $\mathrm{CU}$ in combination with ischaemia assessment with SE, in stable symptomatic women with no history of cardiovascular disease attending for SE.

\section{METHODS}

This was a prospective study in which consecutive women with recent-onset suspected angina who were referred for clinically indicated SE, underwent CU. The principal exclusion criteria included known CAD (including previous coronary revascularisation, previous MI and known $>70 \%$ coronary stenosis), the presence of a resting wall motion abnormality, significant valvular disease, cardiomyopathy, known carotid disease (CD) and prior carotid artery intervention. Clinical data including chest pain character, cardiovascular risk factors, medication and examination findings were gathered. Diabetes was noted if the diagnosis was given in primary care, or if levels of HbAlc were elevated. Hypertension was noted if the diagnosis was given in primary care, or if the patient was on antihypertensive agents. Hypercholesterolaemia was noted if the patient was already on statin therapy or the total cholesterol level was above the reference range. SE data were available for clinical decision-making but not the $\mathrm{CU}$ result, because the latter was analysed 4 months later by a separate reader who did not read the SE, and was blinded to the SE result. Patients with a normal stress echocardiogram were not followed up, and patients with an abnormal result were referred for diagnostic angiography.

\section{Stress echocardiography}

All SE studies were performed using either treadmill exercise or pharmacological (dobutamine-atropine) stress as described previously. ${ }^{9}$ As per protocol heart ratelowering medications were withheld for 48 hours prior to testing. Exercise stress was the preferred modality, and in patients unsuitable for exercise, dobutamine was infused in $3 \mathrm{~min}$ dose increments, starting from $10 \mathrm{mcg} /$ $\mathrm{kg} / \mathrm{min}$ and increasing to 20,30 and $40 \mathrm{mcg} / \mathrm{kg} / \mathrm{min}$. Parasternal long-axis, short-axis and apical four-chamber, two-chamber and three-chamber images were obtained at rest and peak stress (iE33 Philips Medical Systems, Eindhoven, the Netherlands). In patients in whom the endocardial borders of $\geq 2$ contiguous segments were not visualised, the ultrasound contrast agent SonoVue (Bracco, Milan, Italy) was given by intravenous bolus injection $(0.3 \mathrm{~mL})$ and flushed with saline. The final SE result was based on the interpretation of the expert cardiologist (RS). The SEs were reported as normal (normal wall thickening at rest and stress) or ischaemic (inducible wall thickening abnormality $\geq 1$ segment at peak stress).

\section{CU protocol}

B-mode and colour Doppler CU were performed as described previously. ${ }^{10}$ The proximal, mid and distal common carotid artery (CCA), bifurcation of the CCA and proximal portion of the internal and external carotid arteries were systematically interrogated in the long-axis and short-axis views. Plaque was defined as per the Mannheim consensus as a focal structure encroaching into the arterial lumen by $>0.5 \mathrm{~mm}$, a distinct area of intima-media thickness (IMT) $>50 \%$ greater than the adjacent wall or 
$>1.5 \mathrm{~mm}$ in thickness. ${ }^{11}$ IMT measurements were taken at the far wall of the distal CCA at end diastole using a semiautomated edge detection algorithm (Philips Healthcare, Eindhoven, Holland) and the mean value obtained of an average of three readings. $\mathrm{CD}$ was defined as the presence of carotid plaque (CP), or carotid intima-media thickness (CIMT) $>75$ th age and sex-specific percentile. ${ }^{12}$

\section{Coronary angiography}

Standard techniques were used for performing coronary angiography. Images were analysed using a visual quantitative scoring system, with significant $\mathrm{CAD}$ defined as $>70 \%$ luminal diameter narrowing in one or more epicardial coronary arteries or their major branches, since this degree of narrowing is clinically significant.

\section{Assessment of clinical risk}

Framingham Risk Scores (FRS) were calculated with low, intermediate and high risks defined as $<10 \%$, $10 \%-20 \%$ and $>20 \% \quad 10$-year risk of a coronary event, respectively. The pretest probability (PTP) of CAD was calculated based on the UK National Institute for Health and Care Excellence with women being characterised into low $(<10 \%)$, intermediate $(10 \%-90 \%)$ and high $(>90 \%)$ PTP of CAD. ${ }^{13}$

\section{Ascertainment of events}

Data on outcomes were collected by means of a postal questionnaire at 6, 12 and 24 months after the index test, and at the conclusion of the study. The study was concluded after 48 months. Patients gave written consent to be approached for follow-up as part of the study protocol. Patients who did not respond to the postal questionnaire were contacted via telephone. Hospital medical records were also reviewed. Mortality was determined using a national mortality database; a copy of the death certificate was obtained for all deceased patients.

Primary outcome measures were combined major adverse events (MAE), defined as all-cause mortality, nonfatal myocardial infarction (NFMI), heart failure admissions and late coronary revascularisation, with patients censored at the time of event or at the last follow-up. NFMI was defined by the standard criteria of ischaemic chest pain associated with an elevation of cardiac enzymes with or without electrocardiographic changes. Late revascularisation was defined as any revascularisation procedure occurring after 6 months. For patients with multiple events, only the first event was considered.

\section{Statistics}

Categorical variables were expressed as percentages and continuous variables as mean $\pm \mathrm{SD}$. Cox regression analysis was performed to assess the prognostic impact of clinical variables, SE parameters and CU parameters on the time to an MAE. Plaque was tested as both a categorical (present vs absent) and a continuous variable as carotid plaque burden (CPB), based on the number of plaques identified, in separate models. Inducible ischaemia was also tested as a categorical (present vs absent) and a
Table 1 Baseline characteristics of study population

\begin{tabular}{lc}
\hline Patient characteristics & $\mathbf{n}(\%)$ \\
\hline Age & $61(10)$ \\
\hline BMI & $28(5)$ \\
\hline Cardiac risk factors & \\
\hline Hypertension & $208(50)$ \\
\hline High cholesterol & $226(54)$ \\
\hline Diabetes mellitus & $121(29)$ \\
\hline Smoking & $74(18)$ \\
\hline Family history & $184(44)$ \\
\hline PTP & \\
\hline Low (<10) & $58(14)$ \\
\hline Intermediate (10-90) & $328(79)$ \\
\hline High (>90) & $29(7)$ \\
\hline FRS & \\
\hline Low & $208(50)$ \\
\hline Intermediate & $6(2)$ \\
\hline High & $201(48)$ \\
\hline Drugs & \\
\hline Aspirin & $92(22)$ \\
\hline Statin & $143(34)$ \\
\hline BB & $37(9)$ \\
\hline ACEI/ARB & $125(30)$ \\
\hline CCB & $83(20)$ \\
\hline ACEI, ACE
\end{tabular}

ACEI, ACE inhibitor; ARB, angiotensin receptor blocker; BB, beta blocker; BMI, body mass index; CCB, calcium channel blocker; FRS, Framingham Risk Score; PTP, pretest probability.

continuous variable as ischaemic burden, based on the number of ischaemic segments. Patients without an event were censored at the time of last follow-up. Kaplan-Meier survival curves were constructed showing the time to an event and were compared by the log-rank score test. For all tests, a value of $\mathrm{p}<0.05$ was considered statistically significant. All statistical analyses were performed with SPSS V.26.0 (IBM).

\section{RESULTS}

A total of 415 women with a mean age of $61 \pm 10$ years and no history of cardiovascular disease were recruited. The baseline characteristics are shown in table 1 . There was a high prevalence of vascular risk factors-29\% had diabetes mellitus (DM), 50\% had hypertension and 54\% had hypercholesterolaemia. Despite this, $50 \%$ of women were classified as low Framingham risk. The prevalence of women with intermediate PTP was $79 \%$.

In total, 47 women $(11 \%)$ had evidence of inducible myocardial ischaemia. Two hundred and forty-one (58\%) women underwent exercise $\mathrm{SE}$ with the remaining being dobutamine. There were no major complications during SE. 


\begin{tabular}{|c|c|c|c|}
\hline & CD & No CD & $P$ value \\
\hline $\mathrm{n}$ & $190(46)$ & $222(54)$ & \\
\hline Age & 65 & 58 & $<0.001$ \\
\hline \multicolumn{4}{|l|}{ Cardiac risk factors (\%) } \\
\hline Hypertension & $128(67)$ & 81 (36) & $<0.01$ \\
\hline High cholesterol & $109(58)$ & $118(53)$ & 0.43 \\
\hline Diabetes mellitus & $77(41)$ & $46(21)$ & 0.001 \\
\hline Smoker & $38(20)$ & $37(17)$ & 0.48 \\
\hline Family history & $91(48)$ & $97(44)$ & 0.45 \\
\hline Statin & $79(42)$ & $50(22)$ & $<0.001$ \\
\hline PTP & 59 & 41 & $<0.001$ \\
\hline Inducible ischaemia (\%) & $31(16)$ & $16(7)$ & 0.006 \\
\hline BMI & 28.8 & 28.2 & 0.22 \\
\hline \multicolumn{4}{|l|}{ FRS (\%) } \\
\hline Low & $79(42)$ & $125(56)$ & 0.004 \\
\hline Intermediate & $4(2)$ & $3(2)$ & 0.84 \\
\hline High & $107(56)$ & 94 (42) & 0.006 \\
\hline
\end{tabular}

BMI, body mass index; FRS, Framingham Risk Score; PTP, pretest probability.

\section{Carotid disease}

In total, $190(46 \%)$ women had significant CD: CP was present in $170(41 \%)$ and increased CIMT in 62 women $(15 \%)$. CP was identified in the CCA, bifurcation of the CCA and internal carotid artery in $68(40 \%), 135(79 \%)$ and $42(25 \%)$ women. CIMT was not measurable in three patients: in one case due to the extent of plaque, and in two cases due to poor image quality. Table 2 compares the clinical characteristics of patients with and without $\mathrm{CD}$. Women with $\mathrm{CD}$ were older (65 vs 58 years, $\mathrm{p}<0.001$ ), and were more likely to have DM ( $41 \%$ vs $21 \%, \mathrm{p}<0.001)$, hypertension ( $67 \%$ vs $36 \%, \mathrm{p}<0.01$ ), a higher prevalence of high PTP (59\% vs $41 \%, \mathrm{p}<0.001)$ of CAD and higher cardiovascular risk (table 2) as estimated by the FRS.

\section{SE and CU}

All women with inducible ischaemia on SE underwent invasive coronary angiography. Of the 47 women with ischaemia, 24 had flow-limiting disease on coronary angiography, giving a PPV of 51\%. The presence of CP in addition to inducible ischaemia was present in 31 women, of which $22(71 \%)$ had flow-limiting CAD. The PPV was significantly higher compared with those with only ischaemia $(\mathrm{p}<0.01)$. Of the remaining 16 patients with ischaemia but without CP, only two women had flow-limiting disease on angiography, giving a negative predictive value of $88 \%$. Fourteen women $(30 \%)$ with ischaemia had no evidence of $\mathrm{CP}$ or significant CAD. Of the 24 patients with flow-limiting disease and ischaemia, $22(92 \%)$ underwent revascularisation, with two managed medically since intervention was technically challenging.

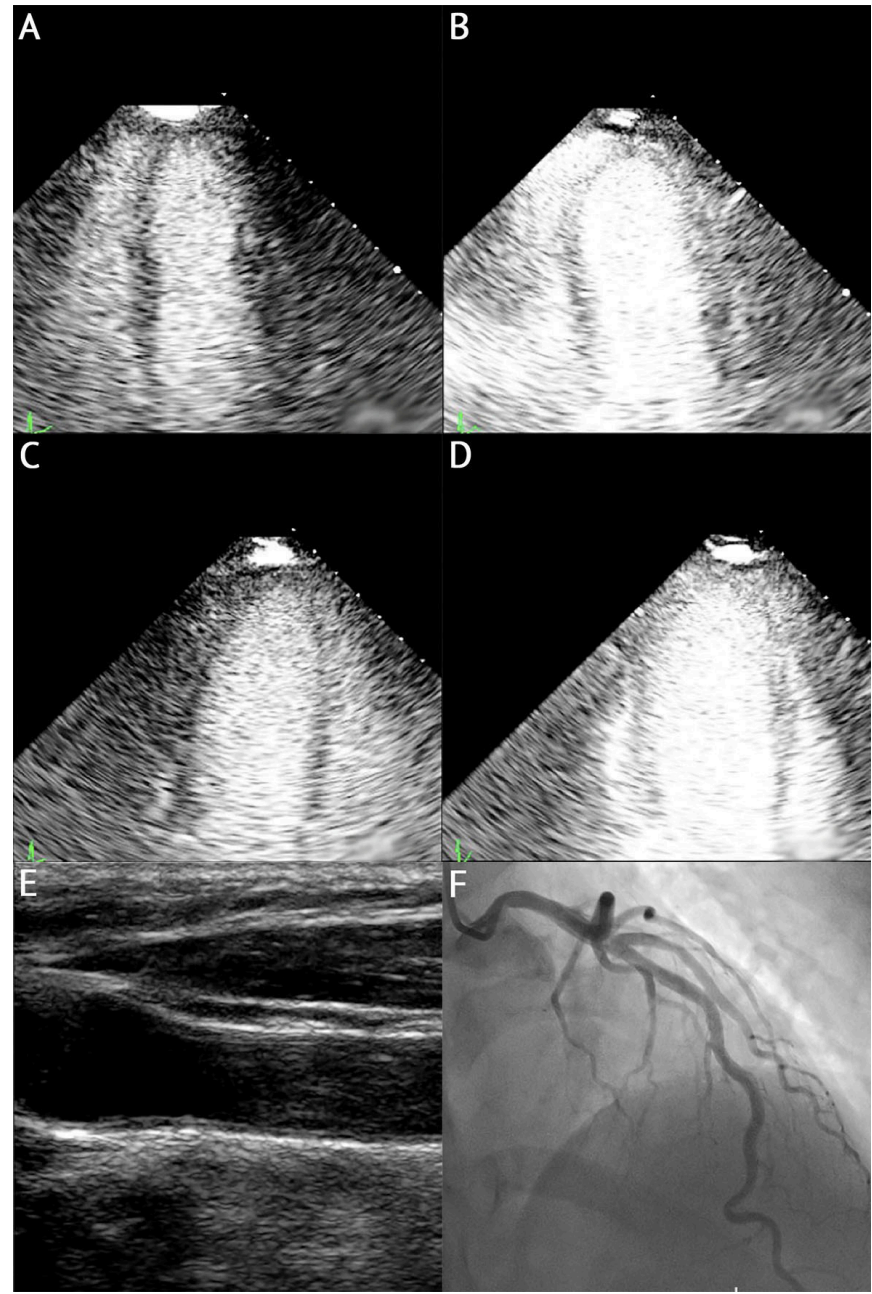

Figure 1 An example of a 59-year-old woman with atypical chest pain and exertional dyspnoea. On exercise echocardiography she exercised for 5.0 metabolic equivalents (METs) and developed left ventricular dilatation at peak stress, with inducible wall motion abnormalities seen in 9/16 wall segments (A-D). Carotid ultrasound (E) and coronary angiography $(F)$ were entirely normal.

Only three patients with negative SE underwent angiography within 6 months for ongoing chest pain symptoms, all of which were negative for flow-limiting CAD.

The mean burden of ischaemia was 4.8 segments and the median was four segments. Ischaemic burden was marginally greater in patients with flow-limiting CAD than in those without (5.2 vs 4.1 segments, $\mathrm{p}=0.04$ ). Figure 1 gives an example of a subject with a large burden of ischaemia and normal CU, with subsequent coronary angiography being normal.

On univariate analysis, age, hypertension, presence of inducible ischaemia and CP predicted the presence of flow-limiting disease. On multivariate analysis, only the presence of inducible ischaemia $(\mathrm{p}=0.01)$ and $\mathrm{CP}$ $(p=0.004)$ were independent predictors.

\section{Reclassification}

In the low and high FRS groups, the prevalence of CD was $42 \%$ and $56 \%$, respectively. Seventy-nine (42\%) 


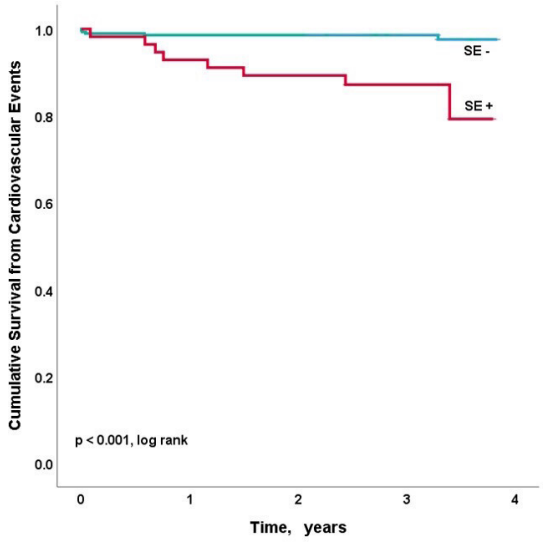

Figure 2 Kaplan-Meier survival curve demonstrating freedom from events based on stress echocardiography result (ischaemia vs no ischaemia). SE, stress echocardiography.

low-risk women were found to have $\mathrm{CD}$. Conversely, 94 $(42 \%)$ patients in the high-risk group had no evidence of CD, 52 of which had DM.

\section{Outcomes}

Of the 415 patients, nine were lost to follow-up. The remaining 406 patients were followed up for a mean interval of $1058 \pm 234$ days. In total, there were 15 events: 3 deaths, 2 admissions with heart failure, 8 admissions with NFMI and 3 late vascularisations (one who later died). The event rate in the cohort was $1.3 \%$ per year.

On univariate Cox regression analysis, age (HR 1.07 (1.00-1.15), $\mathrm{p}=0.04$ ), CPB (HR 1.65 (1.36-2.00), $\mathrm{p}<0.001)$ and ischaemic burden (HR $1.41 \quad(1.18-1.68)$, $\mathrm{p}<0.001)$ were associated with outcome. CIMT did not predict outcome $(p=0.55)$. Since the number of events was modest, multivariate analysis was not performed.

Patients with an abnormal SE had significantly higher event rate compared with normal SE. In patients with normal SE, the event rate was $0.60 \%$ per year vs $4.8 \%$ per year with myocardial ischaemia $(\mathrm{p}<0.001)$ (figure 2$)$. Among the 24 women with ischaemia and flow-limiting CAD, seven had events (event rate 9.7\%/year) and of those 23 women without flow-limiting CAD, two (event rate $2.9 \%$ /year) suffered an event.

Additionally, patients with CP had significantly higher event rates compared with those with no CP. In patients with no $\mathrm{CP}$, the event rate was $0.40 \%$ per year vs $2.3 \%$ per year in those with $\mathrm{CP}(\mathrm{p}=0.001)$ (figure 3$)$.

There was a stepwise increase in events/year from $0.3 \%$ when there were no ischaemia and atherosclerosis versus $1.1 \%$ when there was atherosclerosis and no ischaemia versus $2.2 \%$ when there was ischaemia and no atherosclerosis versus $10 \%$ when there were both ischaemia and atherosclerosis $(p<0.001)$. Figure 4 shows an example of a woman with normal exercise SE and a large burden of echolucent plaque on CU, who presented 3 weeks later with a non-ST elevation MI.

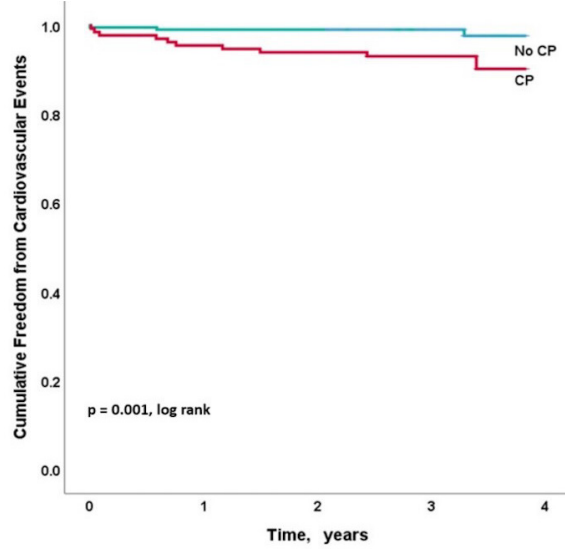

Figure 3 Kaplan-Meier survival curve demonstrating freedom from events based on carotid ultrasound result (carotid plaque vs no plaque). CP, carotid plaque.

\section{DISCUSSION}

This is the first large prospective study in symptomatic women with no history of cardiovascular disease and suspected stable angina, evaluating both atherosclerosis with $\mathrm{CU}$ and myocardial ischaemia with SE. Almost half of women were found to have $\mathrm{CD}$, with a much smaller proportion having evidence of inducible myocardial ischaemia. Inducible ischaemia was predictive of flowlimiting $\mathrm{CAD}$ in only half of the patients with ischaemia, but the presence of CP significantly improved the predictive value of SE to over $70 \%$.

Crucially almost $90 \%$ of women with ischaemia on SE and no evidence of plaque on $\mathrm{CU}$ had no evidence of flow-limiting $\mathrm{CAD}$. Both the presence of $\mathrm{CP}$ and the presence of inducible ischaemia were independent predictors of flow-limiting CAD. Both CPB and ischaemic burden predicted adverse events. However, the absence of both myocardial ischaemia and atherosclerosis was associated with the lowest event rate increasing approximately fourfold when atherosclerosis was seen even in the absence of ischaemia, increasing a further twofold when there was ischaemia but no atherosclerosis, and rising to nearly fivefold when both ischaemia and atherosclerosis were present.

\section{Stress echocardiography}

Almost $50 \%$ of SEs were 'false positive' for obstructive CAD and 14 women (30\%) had symptoms and objective evidence of ischaemia, in the absence of atherosclerosis or CAD, consistent with previous studies. ${ }^{14}$

Ischaemia in the absence of obstructive CAD has a high prevalence in women, as also shown in our study, and coronary microvascular disease (CMD) is a major contributor to this paradox. ${ }^{12}$ In the present study, the event rate in women with myocardial ischaemia and non-obstructive disease was substantial-almost 3\% per year-which was approximately 2.5 times the prevalent event rate in the population. It is well recognised that myocardial ischaemia detected by SE is associated with subsequent IHD 


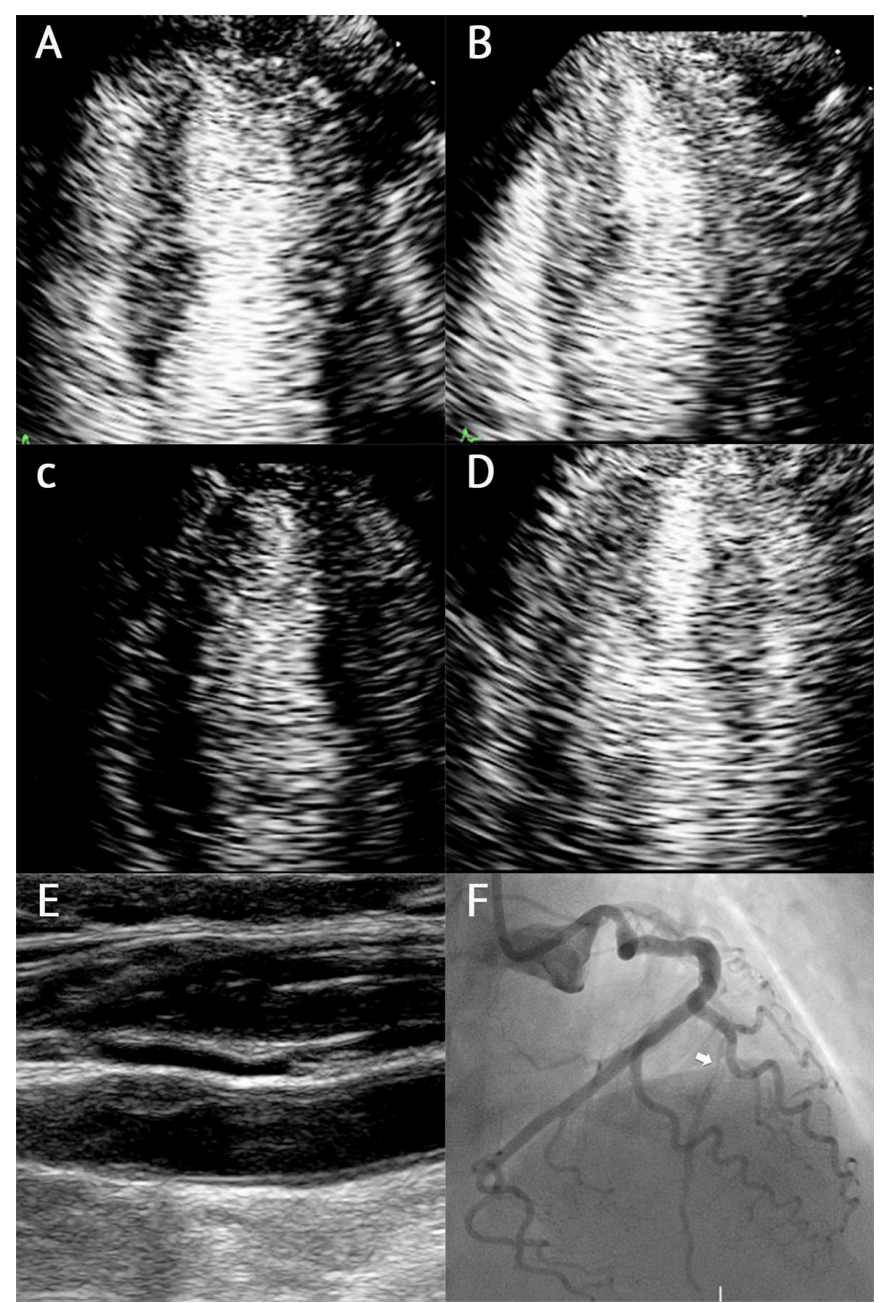

Figure 4 A 55-year-old woman presented with left-sided chest pains. Exercise echocardiography was normal (A-D) and carotid ultrasound (CU) demonstrated a large burden of echolucent plaque in the carotid bulb (E). She presented 3 weeks later with a non-ST elevation myocardial infarction and angiography (F) demonstrated a subtotal occlusion of the proximal left anterior descending coronary artery (LAD) with heavy thrombus burden (white arrow) which was stented.

events, even in the absence of obstructive CAD on angiography. $^{1516}$

There is close association of cardiovascular risk factors and atherosclerosis in the majority of patients with CMD. ${ }^{17}$ Thus, aggressive management of comorbidities is an important goal of treatment in CMD. This includes smoking cessation, weight loss, adequate control of blood pressure, diabetes and related metabolic abnormalities, lipid management, improved nutrition and regular exercise. ${ }^{17} \mathrm{~A}$ paired stress imaging study with interval anti-ischaemic and preventative medications may prove beneficial in women with ischaemia and non-obstructive $\mathrm{CAD}$, and is currently under investigation, as are alternative therapeutic strategies. ${ }^{18} 19$

Although women with a negative stress echocardiogram did not undergo angiography, their event rate was very low, suggesting a very low prevalence of prognostically significant CAD. SE was therefore able to differentiate high-risk patients (event rate $\sim 5 \%$ /year) from low-risk patients (event rate $\sim 0.5 \%$ /year) as expected, extending its excellent risk stratification to women.

\section{CU and traditional risk markers}

The FRS is used to determine an individual's 10-year risk of CAD, death or MI to guide therapeutic intervention for both low-density lipoprotein cholesterol and hypertension. ${ }^{20}$ Patients at greatest risk should receive aggressive therapeutic and lifestyle recommendations. However, the FRS classifies $>90 \%$ of women as low risk. ${ }^{21}$

In this study, just over $40 \%$ of patients with low FRS had CD and almost one-fifth of the study population would have benefited from primary prevention therapy that were deemed low risk. In the BioImage study, ${ }^{22}$ where the mean age was 69 years and $57 \%$ were female, this proportion was $50 \%$, and CPB improved the net reclassification index for events. In a cross-sectional study of 2447 non-diabetic women, $84 \%$ of those with significant coronary artery calcification (CAC) were classified with a low FRS. $^{23}$ These findings highlight the imprecision of FRS estimates in women, the prevalent, undetected burden of atherosclerosis in females and the clinical utility of atherosclerosis imaging. Further studies should evaluate whether therapeutic intervention improves outcomes.

As hypothesised, the presence of CP increased the PPV of inducible ischaemia for predicting flow-limiting CAD. Since non-obstructive atherosclerosis is a major cause of ischaemia in women, ${ }^{24}$ it is not surprising that the PPV of $\mathrm{SE} / \mathrm{CU}$ was close to $70 \%$. In a study of CU and CT angiography, the prevalence of $\mathrm{CD}$ in patients with and without obstructive disease was $70 \%$ and $43 \%$, respectively. ${ }^{7}$

$\mathrm{CP}$ presence (CPB) and ischaemia were strong independent predictors of outcome. Although atherosclerosis precedes the development of obstructive CAD, which manifests clinically as ischaemia, most acute coronary syndromes occur secondary to plaque rupture in patients with mild atherosclerosis. ${ }^{6}$ Conversely ischaemia may occur in the absence of atherosclerosis and cause cardiac events. ${ }^{24}$ The data on the synergistic benefit of atherosclerosis and myocardial ischaemia have been previously confined to patients undergoing CT/single-photon emission CT (SPECT) or CT/positron emission tomography (PET), ${ }^{25}$ but we have clearly shown that it is applicable to cardiovascular ultrasound in the present setting.

\section{Ultrasound as the technique of choice in symptomatic women} The diagnostic and prognostic value of combined SE and CU has been previously demonstrated in a mixed cohort of men and women with symptoms suggestive of stable angina. ${ }^{8} 10$ This study extends the diagnostic and prognostic benefit to women.

The National Council for Radiation Protection and Measurement has emphasised several key principles to guide referral of women for non-invasive testing, which include an emphasis on justification of use, dose reduction optimisation and an adequate knowledge base to guide use. ${ }^{4}$ Only $12 \%$ of women underwent angiography 
here, despite securing a low event rate of $1.3 \% /$ year. The low event rates in women with no ischaemia and no CP make it difficult to justify subjecting women to more expensive tests, which require exposure to ionising radiation (such as SPECT/PET, CT/invasive angiography). Echocardiography also has the advantages of being able to detect non-coronary cardiac conditions in women contributing to exercise intolerance such as diastolic dysfunction, elevated pulmonary pressures and the subtle detection of abnormal myocardial mechanics through deformation imaging at rest and stress. 3D imaging among other things allows tomographic assessment of the left ventricle.

Both CD and coronary artery calcium score are imaging biomarkers of subclinical atherosclerosis, and are both comparable for predicting events in older populations. ${ }^{22}$ However, there are concerns in younger patients and women who may have non-calcified high-risk plaque which have not yet developed calcification. In a study of asymptomatic women, $55 \%$ of patients with a CAC of 0 had $\mathrm{CD},{ }^{26}$ and in a younger, mixed gender cohort this was $47 \%{ }^{27}$

Since CU is likely to be more cost-effective and lacks radiation, it may be more suitable for longitudinal lifelong surveillance if no plaque burden is identified at baseline and may be a first-line test in younger individuals and in women in whom plaque calcification may not have developed, although this needs to be evaluated in further studies.

\section{Clinical implication}

This study has confirmed our hypotheses for the role of $\mathrm{CU}$ in women attending for SE. The number of falsepositive tests was almost halved, over one-third of women required secondary prevention not indicated by the FRS and patients with no $\mathrm{CP}$ or ischaemia had a very low event rate. Conversely women with $\mathrm{CP}$ or ischaemia had intermediate to high event rates.

We have suggested a multimodality algorithm for women with suspected CAD to minimise ionising radiation (figure 5). Women with ischaemia and CP should undergo invasive coronary angiography as the probability of flow-limiting CAD is above $70 \%$. On the other hand, in women with ischaemia and no evidence of carotid atherosclerosis the prevalence of flow-limiting CAD is low, around $10 \%$, and these patients may proceed to relatively low-risk Computerised tomography coronary angiography (CCTA). Women with no evidence of ischaemia but presence of carotid atherosclerosis may be given primary preventative therapy in view of fourfold increase in event rates compared with those patients without atherosclerosis and ischaemia. The latter group only needs reassurance as their prognosis is excellent.

In persistently symptomatic women with ischaemia and non-obstructive $\mathrm{CAD}$, the assessment of coronary flow reserve (CFR) invasively or non-invasively (depending on the angiogram type) may be indicated to identify $\mathrm{CMD}$, and guide potential therapeutic strategies, and prognosis. CMD can be defined as impaired CFR in the absence of obstructive $\mathrm{CAD}$, reflecting downstream vasomotor dysfunction. Non-invasive techniques to identify CMD include PET, Doppler echocardiography, myocardial contrast echocardiography and cardiac magnetic resonance.

\section{Limitations}

Since the follow-up was relatively short, the number of events was relatively low. Our study was carried out in

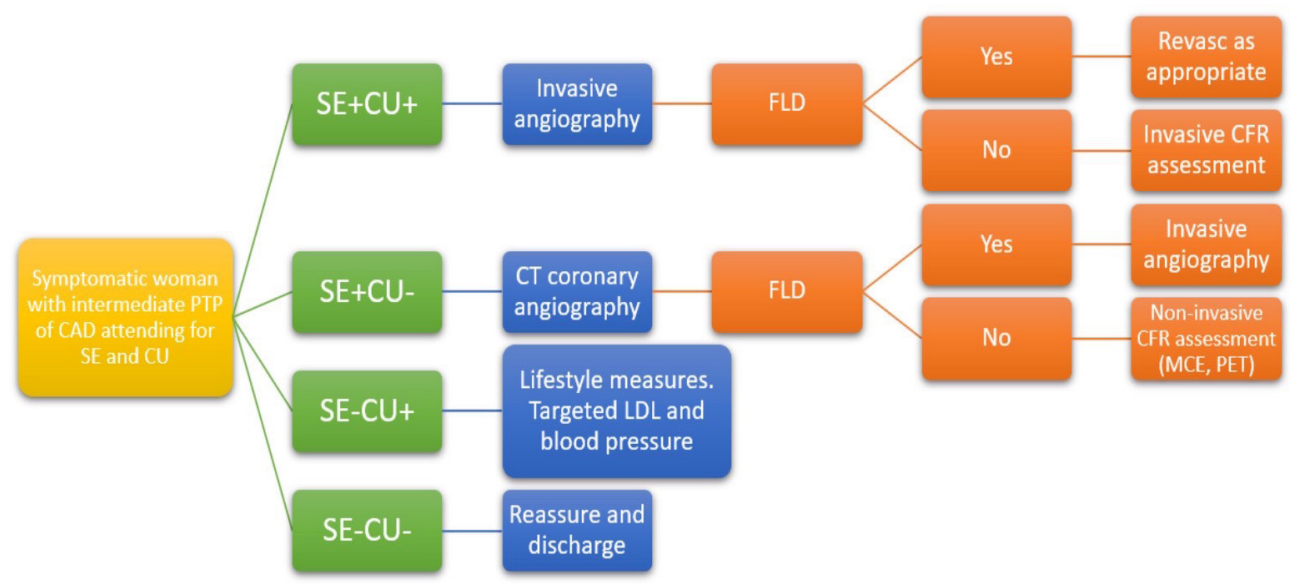

Figure 5 A suggested multimodality algorithm in women with suspected coronary artery disease (CAD). Women with ischaemia and carotid plaque (CP) should undergo invasive coronary angiography since the probability of flow-limiting disease (FLD) is $70 \%$. In contrast, women with ischaemia and no CP may undergo CT coronary angiography since the prevalence of FLD is only around $10 \%$. Where FLD is not demonstrated, assessment of coronary flow reserve (CFR) should be performed invasively or non-invasively using myocardial contrast echocardiography (MCE), Doppler echocardiography, cardiac magnetic resonance (CMR) or positron emission tomography (PET). Women with no ischaemia and CP may benefit from aggressive risk factor modification and lifestyle measures. Where there is no ischaemia or CP, women can be safely discharged since the prognosis is excellent. CU, carotid ultrasound; LDL, low-density lipoprotein; PTP, pretest probability; SE, stress echocardiography. 
a single, high-volume centre with a single but different reader interpreting the $\mathrm{SE}$ and $\mathrm{CU}$ studies. However, multiple operators performed both SE and CU. These findings ideally should be replicated in a multicentre study. CFR was not performed in this study, and further studies should explore the link between CPB, CFR, ischaemia and events in women. The study did not assess the role of primary prevention therapy in patients with $\mathrm{CP}$ and no ischaemia. Thus, no firm conclusions can be drawn regarding the outcome of primary preventative therapy in this group of patients.

\section{CONCLUSION}

Besides identifying atherosclerosis in a large proportion of low-risk women, CU significantly improved the PPV of inducible wall motion abnormalities on SE. Women with no evidence of inducible ischaemia or atherosclerosis had very low event rates, making this combination imaging an excellent screening test in women. Because women are a relatively low-risk population, low-risk efficacious procedures like SE and CU should be the first-line test in symptomatic women with suspected CAD, in highvolume centres.

\section{Twitter Sothinathan Gurunathan @DrNathan001}

Contributors RS and SG conceived and designed the study. Data were acquired by SG, MS and RH. SG and MS drafted the manuscript, which was critically revised by RK and RS. All authors approved the final version of the manuscript.

Funding The study was supported by Northwick Park Cardiac Research Fund.

Competing interests RS has received speaker fees from Bracco (Italy), Phillips (Netherlands) and Lantheus Medical Imaging.

Patient consent for publication Not required.

Ethics approval Ethical approval for the study was obtained by London City and East REC Ethics Committee (REC reference 14/L0/1736).

Provenance and peer review Not commissioned; externally peer reviewed.

Data availability statement Data are available upon reasonable request. Data are available from Northwick Park Cardiac Research Charity.

Open access This is an open access article distributed in accordance with the Creative Commons Attribution Non Commercial (CC BY-NC 4.0) license, which permits others to distribute, remix, adapt, build upon this work non-commercially, and license their derivative works on different terms, provided the original work is properly cited, appropriate credit is given, any changes made indicated, and the use is non-commercial. See: http://creativecommons.org/licenses/by-nc/4.0/.

\section{ORCID IDs}

Sothinathan Gurunathan http://orcid.org/0000-0001-7143-0655

Roxy Senior http://orcid.org/0000-0001-6579-7039

\section{REFERENCES}

1 WHO. Global health Observatory (GHO) data. Available: https://www. who.int/gho/women_and health/en/

2 Mozaffarian D, Benjamin EJ, Go AS, et al. Heart disease and stroke statistics--2015 update: a report from the American Heart Association. Circulation 2015;131:e29-322.

3 Shaw LJ, Merz CNB, Pepine CJ, et al. The economic burden of angina in women with suspected ischemic heart disease: results from the National Institutes of Health--National Heart, Lung, and Blood Institute--sponsored Women's Ischemia Syndrome Evaluation. Circulation 2006;114:894-904.

4 Mieres JH, Shaw LJ, Arai A, Gulati M, Bairey Merz N, et al. Role of noninvasive testing in the clinical evaluation of women with suspected coronary artery disease: consensus statement from the cardiac imaging Committee, Council on clinical cardiology, and the cardiovascular imaging and intervention Committee, Council on cardiovascular radiology and intervention, American heart association. Circulation 2005;111:350-79.

5 Shaw esleeJ, Gibbons RJ, McCallister B, et al. Gender differences in extent and severity of coronary disease in the ACC national cardiovascular disease registry. J Am Coll Cardiol 2002;39: 321.

6 Stone GW, Maehara A, Lansky AJ, et al. A prospective naturalhistory study of coronary atherosclerosis. N Engl J Med 2011;364:226-35.

7 Guaricci Al, Pontone G, Fusini L, et al. Additional value of inflammatory biomarkers and carotid artery disease in prediction of significant coronary artery disease as assessed by coronary computed tomography angiography. Eur Heart J Cardiovasc Imaging 2017;18:1049-56.

8 Ahmadvazir S, Shah BN, Zacharias K, et al. Incremental Prognostic Value of Stress Echocardiography With Carotid Ultrasound for Suspected CAD. JACC Cardiovasc Imaging 2018;11: 173-80.

9 Gurunathan S, Zacharias K, Akhtar M, et al. Cost-Effectiveness of a management strategy based on exercise echocardiography versus exercise electrocardiography in patients presenting with suspected angina during long term follow up: a randomized study. Int $J$ Cardiol 2018;259:1-7.

10 Ahmadvazir S, Zacharias K, Shah BN, et al. Role of simultaneous carotid ultrasound in patients undergoing stress echocardiography for assessment of chest pain with no previous history of coronary artery disease. Am Heart J 2014;168:229-36.

11 Touboul P-J, Hennerici MG, Meairs S, et al. Mannheim carotid intima-media thickness and plaque consensus (2004-20062011). An update on behalf of the Advisory Board of the 3rd, 4th and 5th watching the risk symposia, at the 13th, 15th and 20th European stroke conferences, Mannheim, Germany, 2004, Brussels, Belgium, 2006, and Hamburg, Germany, 2011. Cerebrovasc Dis 2012;34:290-6.

12 Stein JH, Korcarz CE, Hurst RT, et al. Use of carotid ultrasound to identify subclinical vascular disease and evaluate cardiovascular disease risk: a consensus statement from the American Society of echocardiography carotid intima-media thickness Task force endorsed by the Society for vascular medicine. J Am Soc Echocardiogr 2008;21:93-111.

13 Cooper A, Timmis A, Skinner J, et al. Assessment of recent onset chest pain or discomfort of suspected cardiac origin: summary of NICE guidance. BMJ 2010;340:c1118.

14 Khaliq A, Johnson BD, Anderson RD, et al. Relationships between components of metabolic syndrome and coronary intravascular ultrasound atherosclerosis measures in women without obstructive coronary artery disease: the NHLBI-Sponsored women's ischemia syndrome evaluation study. Cardiovasc Endocrinol 2015;4:45-52.

15 From AM, Kane G, Bruce C, et al. Characteristics and outcomes of patients with abnormal stress echocardiograms and angiographically mild coronary artery disease ( $<50 \%$ stenoses) or normal coronary arteries. J Am Soc Echocardiogr 2010;23:207-14.

16 Gulati M, Cooper-DeHoff RM, McClure C, et al. Adverse cardiovascular outcomes in women with nonobstructive coronary artery disease: a report from the women's ischemia syndrome evaluation study and the ST James women take heart project. Arch Intern Med 2009;169:843-50.

17 Taqueti VR, Di Carli MF. Coronary Microvascular Disease Pathogenic Mechanisms and Therapeutic Options: JACC State-of-the-Art Review. J Am Coll Cardiol 2018;72:2625-41.

18 Bairey Merz CN, Pepine CJ, Walsh MN, et al. Ischemia and NO obstructive coronary artery disease (INOCA): developing evidencebased therapies and research agenda for the next decade. Circulation 2017;135:1075-92.

19 . Available: https://www.ischemiatrial.org/ciao-ischemia

20 Wilson PW, D'Agostino RB, Levy D, et al. Prediction of coronary heart disease using risk factor categories. Circulation 1998;97:1837-47.

21 Pasternak RC, Abrams J, Greenland P, et al. 34th Bethesda Conference: Task force \#1--Identification of coronary heart disease risk: is there a detection gap? J Am Coll Cardiol 2003;41:1863-74.

22 Baber U, Mehran R, Sartori S, et al. Prevalence, impact, and predictive value of detecting subclinical coronary and carotid atherosclerosis in asymptomatic adults: the Biolmage study. J Am Coll Cardiol 2015;65:1065-74.

23 Michos ED, Nasir K, Braunstein JB, et al. Framingham risk equation underestimates subclinical atherosclerosis risk in asymptomatic women. Atherosclerosis 2006;184:201-6.

24 Taqueti VR, Shaw LJ, Cook NR, et al. Excess cardiovascular risk in women relative to men referred for coronary angiography 
is associated with severely impaired coronary flow reserve, not obstructive disease. Circulation 2017;135:566-77.

25 van Werkhoven JM, Schuijf JD, Gaemperli O, et al. Prognostic value of multislice computed tomography and gated single-photon emission computed tomography in patients with suspected coronary artery disease. J Am Coll Cardiol 2009;53:623-32.
26 Roy RR, Hurst RT, Lester SJ, et al. Risk Stratification for Cardiovascular Disease in Women in the Primary Care Setting. J Am Soc Echocardiogr 2015;28:1232-9.

27 Cohen Gl, Aboufakher R, Bess R, et al. Relationship between carotid disease on ultrasound and coronary disease on CT angiography. JACC Cardiovasc Imaging 2013;6:1160-7. 